\title{
Candidate genes for shell colour polymorphism in Cepaea nemoralis
}

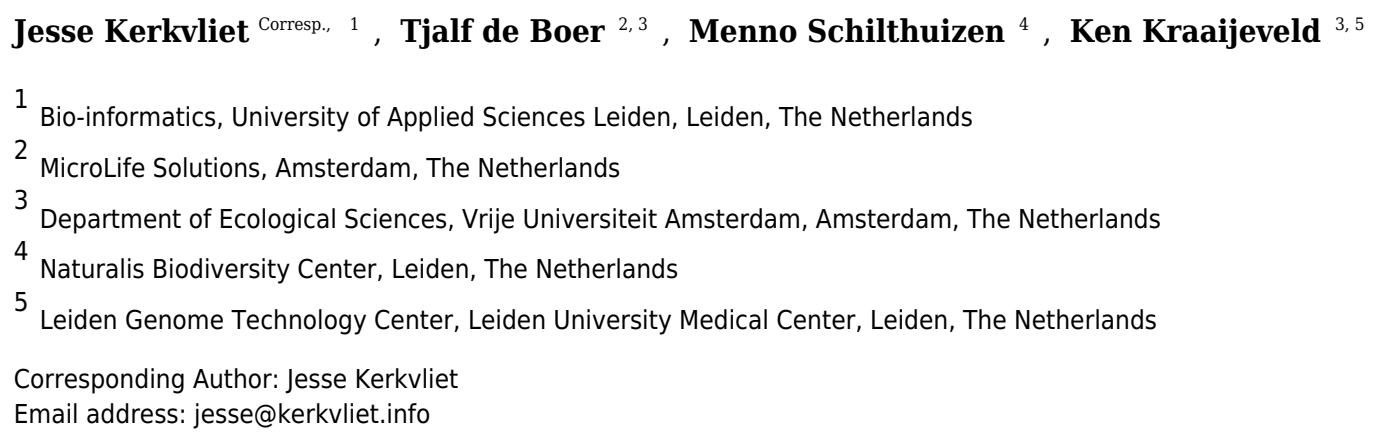

The characteristic ground colour and banding patterns on shells of the land snail Cepaea nemoralis form a classic study system for genetics and adaptation as it varies widely between individuals. We use RNAseq analysis to identify candidate genes underlying this polymorphism. We sequenced cDNA from the foot and the mantle (the shell-producing tissue) of four individuals of two phenotypes and produced a de novo transcriptome of 147,397 contigs. Differential expression analysis identified a set of 1,961 transcripts that were upregulated in mantle tissue. Sequence variant analysis resulted in a set of 2,592 transcripts with single nucleotide polymorphisms (SNPs) that differed consistently between the phenotypes. Inspection of the overlap between the differential expression analysis and SNP analysis yielded a set of 197 candidate transcripts, of which 38 were annotated. Four of these transcripts are thought to be involved in production of the shell's nacreous layer. Comparison with morph-associated Restriction-site Associated DNA (RAD)-tags from a published study yielded eight transcripts that were annotated as metallothionein, a protein that is thought to inhibit the production of melanin in melanocytes. These results thus provide an excellent starting point for the elucidation of the genetic regulation of the Cepaea nemoralis shell colour polymorphism. 


\section{Candidate genes for shell colour polymorphism in Cepaea nemoralis}

2

3 Jesse Kerkvliet ${ }^{1}$, Tjalf de Boer ${ }^{2,3}$, Menno Schilthuizen ${ }^{4}$, Ken Kraaijeveld ${ }^{2,5}$

4

$5{ }^{1}$ Bioinformatics, University of Applied Sciences Leiden, Leiden, The Netherlands

$6{ }^{2}$ Department of Ecological Sciences, Vrije Universiteit Amsterdam, Amsterdam, The

7 Netherlands

$8{ }^{3}$ MicroLife Solutions, Amsterdam, The Netherlands

$9 \quad{ }^{4}$ Naturalis Biodiversity Center, Leiden, the Netherlands

$10{ }^{5}$ Leiden Genome Technology Center, Leiden University Medical Center, Leiden, The

11 Netherlands

12

13 Corresponding author: Jesse Kerkvliet, Jesse@Kerkvliet.info

14

15

16 


\section{Abstract}

18 The characteristic ground colour and banding patterns on shells of the land snail Cepaea

19 nemoralis form a classic study system for genetics and adaptation as it varies widely

20 between individuals. We use RNAseq analysis to identify candidate genes underlying this

21 polymorphism. We sequenced cDNA from the foot and the mantle (the shell-producing

22 tissue) of four individuals of two phenotypes and produced a de novo transcriptome of

23147,397 contigs. Differential expression analysis identified a set of 1,961 transcripts that

24 were upregulated in mantle tissue. Sequence variant analysis resulted in a set of 2,592

25 transcripts with single nucleotide polymorphisms (SNPs) that differed consistently

26 between the phenotypes. Inspection of the overlap between the differential expression

27 analysis and SNP analysis yielded a set of 197 candidate transcripts, of which 38 were

28 annotated. Four of these transcripts are thought to be involved in production of the shell's

29 nacreous layer. Comparison with morph-associated Restriction-site Associated DNA (RAD)-

30 tags from a published study yielded eight transcripts that were annotated as

31 metallothionein, a protein that is thought to inhibit the production of melanin in

32 melanocytes. These results thus provide an excellent starting point for the elucidation of

33 the genetic regulation of the Cepaea nemoralis shell colour polymorphism. 


\section{Introduction}

38 Since the first studies of selection on the banding patterns and colours on the shell of the

39 land snail Cepaea nemoralis over 60 years ago (Cain \& Sheppard, 1950, 1952, 1954), the

40 polymorphism has become a classic study system for genetics and adaptation (Jones, Leith

41 \& Rawlings, 1977; Cook, 1998; Silvertown et al., 2011; Cameron \& Cook, 2012). For

42 example, shell colour affects habitat-dependent predation risk and thermoregulation

43 (Lamotte, 1959; Clarke, 1962; Arnold, 1971; Greenwood, 1974). More recently, the Cepaea

44 shell colour polymorphism became the subject of the citizen science project Evolution

45 MegaLab, in which citizen scientists are asked to score the phenotypes of Cepaea snails in

46 their surroundings and upload their records to the MegaLab website (Worthington et al.,

47 2012). The aim of this project is to show that (possibly human-induced) selection

48 differences can result in differences in allele frequencies on a local as well as a continental

49 scale (Silvertown et al., 2011; Schilthuizen, 2013).

50

51 Despite the prominence of the Cepaea study system in scientific discourse as well as public 52 outreach, little is known about the underlying molecular genetic machinery that produces

53 the different phenotypes. The Cepaea shell polymorphism consists of nine phenotypic

54 traits, which include shell ground colour and various aspects of the banding pattern

55 (Richards et al., 2013). Genes underlying five of these traits are closely linked to form a so-

56 called supergene (Schwander, Libbrecht \& Keller, 2014), which inherit with very little

57 recombination between the alleles, keeping the alleles together as one large gene.

58 (Schwander, Libbrecht \& Keller, 2014). Richards et al. (2013) identified eleven restriction-

59 site associated DNA (RAD) tags that were linked to the supergene. Three of these tags were 
60 within $\sim 0.6 \mathrm{cM}$ of the supergene. Mann \& Jackson (2014) characterized the major

61 proteinaceous components of the $C$. nemoralis shell, but were unable to identify proteins

62 associated with shell pigmentation. The aim of our work is to identify candidate genes that

63 may underlie the Cepaea shell polymorphism.

64

65 To identify candidate genes that play a role in the polymorphism, the RNA of four juvenile

66 C. nemoralis individuals (two brown/unbanded and two yellow/banded) was sequenced.

67 For each individual, we sequenced the transcriptome of the mantle (the organ in which the

68 shell is formed) and the snail foot. Since the polymorphism is only visible in the shell, we

69 focus on candidate genes that are upregulated in the mantle. Within this set of mantle-

70 specific genes, we search for sequence variants that differ consistently between different

71 phenotypes. Furthermore, we search for the three supergene-associated RAD tags reported

72 by (Richards et al., 2013), as they are found in near proximity of the supergene. Our results

73 provide the first clues to the molecular mechanisms underlying the Cepaea polymorphism

74 and will provide a starting point for elucidating the genetic architecture of this classic

75 polymorphism.

76

77 Methods

78 Sample collection, $m R N A$ extraction, sequencing and assembly

79 Four juvenile $C$. nemoralis with different phenotypes (two with brown shell and no banding

80 and two with a yellow shell with multiple dark bands; Fig. S1) were collected at the Van

81 Veldhuizenbos near Dronten, The Netherlands. Total RNA was extracted separately from

82 the mantle and the foot for each of the four C. nemoralis individuals using the NucleoSpin 
83 RNA kit (Macherey-Nagel), following the manufacturer's protocol. The remains of the

84 specimens have been deposited in the alcohol collection of Naturalis Biodiversity Center

85 under reference numbers RMNH.5004228-5004231. The total RNA was subjected to polyA

86 selection, converted to cDNA and used to generate sequencing libraries as described in

87 (Salazar-Jaramillo et al., 2017). The libraries were paired-end 2x100 bp sequenced on an

88 Illumina HiSeq 2000 at the Leiden Genome Technology Center.

89

90

91

92

93

03

De novo assembly, protein prediction and annotation

Sequence reads of all eight samples were combined to create a reference transcriptome assembly using Trinity v2.0.5 with default settings (Grabherr et al., 2011). To reduce redundancy, transcripts were clustered using CD-HIT-Est (Fu et al., 2012) at a cut-off of 95\% identity. To remove contamination, we used NCBI's VecScreen. This tool searches for segments that match sequences in the UniVec database. We used BUSCO (Simao et al., 2015) to assess the completeness of the transcriptome. This tool searches the transcriptome for a reference set of single-copy orthologs. The metazoan set (odb9) was used as a reference set. All genes in the transcriptome were annotated for gene ontology (GO) using the Trinotate part of the Trinity package. Trinotate uses blastp and blastx to compare the predicted peptide sequences and the reference transcriptome to the uniprot_sprot and the uniprot_uniref peptide databases, performs an hmm-scan on the Pfam-A database and assigns GO terms.

Differential expression 
105 To identify genes that were overexpressed in the shell-forming mantle tissue, the Trinity

106 RNA-seq pipeline (Grabherr et al., 2011) was used. First, the reads for both organs in all

107 four individuals were mapped to the reference transcriptome. Second, the mapped reads

108 were counted to visualize the expression of these transcripts, using the estimation method

109 eXpress (Roberts \& Pachter, 2013). A high count of mapped reads indicates a high

110 expression rate, while a low count of mapped reads indicates a low expression rate. With

111 the estimated counts of reads, expression profiles were generated using the R-package

112 EdgeR (Robinson, McCarthy \& Smyth, 2010). Normalization took place as part of the EdgeR

113 workflow. The profiles were then filtered on fold change and false discovery rate (FDR)

114 with the Trinity default cut-off scores of 4 and 0.001 respectively. The genes that were

115 overexpressed in the mantle were selected for further analysis. A heatmap of differentially

116 expressed genes versus samples was produced using the script analyze_diff_expr.pl within

117 the Trinity package.

119 Sequence variants

120 To identify consistent sequence differences between the two phenotypes (yellow/banded

121 and the brown/unbanded), we conducted variant calling following the following protocol.

122 First, reads were mapped to the reference transcriptome using Bowtie2 (Langmead \&

123 Salzberg, 2012). Next, GATK's HaplotypeCaller (McKenna et al., 2010) was run to search for

124 single-nucleotide polymorphisms (SNPs). The default parameters were used with a

125 stand_call confidence of 20.0 and a stand_emit confidence of 20.0. The VariantFiltration

126 tool was used with default parameters to filter out false-positive variants. Clusters were

127 generated when 3 SNPs were present within a window of 35 bases. Variants with a Fisher- 
128 strand Score (FS) greater than 30.0 or a quality by depth (QD) value less than 2.0 were

129 filtered out. SNPs with sequencing depth less than 10 in any of the samples were removed.

130 The effect of each SNP on the transcript product was predicted using SnpEff (Cingolani et

131 al., 2012).

132 The resulting set of SNPs was filtered for consistency with the phenotypes using

133 GATK and custom R-scripts. The inheritance of the polymorphism is well understood (Cain

134 \& Sheppard, 1954; Murray, 1963; Jones, Leith \& Rawlings, 1977). The brown ground colour

135 is dominant over the yellow ground colour and absence of banding is dominant over the

136 presence of multiple bands. The candidate SNPs were therefore filtered so that the brown,

137 unbanded snails were either heterozygous or homozygous and the yellow, banded snails

138 were homozygous. Mantle-enriched transcripts contained at least one phenotype-

139 consistent sequence variant were blastn-searched (Altschul et al., 1990) against the non-

140 redundant nucleotide database.

141

142 RAD-tags

143 Richards et al. (2013) identified eleven anonymous RAD tag sequences that lie near the

144 colour-polymorphism supergene. In the original research, a cut-off of 96 base pairs was

145 used for the tags. Because of this, the tags are shorter than the full assembly of the RAD tag

146 reads. The overlapping parts of the RAD tag reads were used to generate a consensus for

147 each tag that was longer than the original consensus RAD tag. These extended tags were

148 blast-searched against the reference transcriptome using the megablast algorithm within

149 the Blast+ (Camacho et al., 2009) command line tool. Hits were filtered by E-value less than $15010^{-10}$. 
152 Results

\section{Transcriptome assembly and annotation}

154 Eight RNAseq libraries (two for each individual) were constructed and sequenced, yielding 155 between $63.9 \times 10^{6}$ and $139.5 \times 10^{6}$ paired reads per sample (Table 1 ). These reads were 156 assembled into contigs representing 171,051 putative transcripts deriving from 33,109 157 putative genes (Table 2). The frequency of the number of transcripts per gene is shown in 158 Fig. S2. Clustering using CD-HIT reduced the number of transcripts to 150,380. On average, $15971 \%$ of the raw sequence reads aligned at least once to the reference transcriptome (Table 160 S1). After removing vector contamination, 147,397 transcripts remained.

161 The completeness of the transcriptome was assessed using BUSCO. Of the 978 162 single-copy orthologs that were searched for, 920 were found completely. Of these, 765 163 (78.2\%) were found single-copy, 155 (15.8\%) orthologs were found duplicated. A further $16437(3.8 \%)$ of the orthologs were found fragmented and 21 (2.2\%) were not found in the 165 transcriptome.

167 sequence comparison to the uniprot_sprot database yielded annotations for 89,386(60.6\%) 168 transcripts, to the uniprot_uniref database for 87,045 (59.1\%) transcripts and to the Pfam 169 database for 30,405 (20.6\%) transcripts. After annotation, Trinotate assigned GO-terms to 17019,658 genes, covering 26,039 transcripts (17.7\%; Data S1).

171

172

Differential expression 
173 Hierarchical clustering of the overall expression data clearly separated the mantle and foot

174 tissue samples (Fig. 1). EdgeR identified 1,961 transcripts as upregulated and 1,260 as

175 downregulated in the mantle relative to the foot (Fig. 2, Data S2).

176

177 Sequence polymorphisms

178 A total of 73,817 SNPs passed our filtering steps (Table S2). This included 12,273

179 synonymous and 12,451non-synonymous variants. The remaining 49,092 SNPs fell outside 180 predicted open reading frames. A total of 569 SNPs were found in transcripts that were 181 overexpressed in the mantle and showed phenotype-consistent allelic variation (Table 3).

182 A total of 197 mantle-enriched transcripts contained at least one phenotype-consistent

183 sequence variant. This set was subjected to more detailed annotation. We obtained 184 database matches for 98 transcripts, of which 38 were functionally annotated (Table S3).

185 Annotations that indicate putative functions related to shell formation are summarized in 186 Table 4. Prominent among these annotations are sequences that are thought to play a role

187 in the production of the nacreous layer in mollusc shells, including dermatopin and 188 nacroperlin genes (marked in Table 4).

189

190

$R A D$-tags

191 A set of 12 transcripts had a match with at least one of the elongated tags that lie in close 192 proximity to the supergene (Richards et al., 2013) (Table S4). None of these transcripts

193 were found in our list of differentially expressed transcripts with phenotype-consistent 194 variants. Eight of the twelve transcripts (matching two of the RAD tags closest to the 195 supergene) had a blast hit to the nr and nt databases (Table S4, Data S3). All of these hits 
196 were the Helix pomatia homolog of metallothionein. This same sequence was also found in

197 the variant analysis (six of 197 transcripts $=3 \%$ ). None of the transcripts in these two sets

198 overlap. The percentage of metallothionein hits among the entire reference transcriptome

199 was $6,1 \%(2,506$ of 40,748 transcripts $)$, suggesting that this gene is not overrepresented in 200 our variant analysis.

201

\section{Discussion}

203 Our analysis identified a list of 300 candidate transcripts that were differentially expressed 204 in the shell-forming mantle tissue and contained SNP patterns that matched the shell 205 phenotypes. For most of these transcripts, it was impossible to infer their role in shell 206 formation as only $\sim 20 \%$ of these genes were functionally annotated. Furthermore, the 207 supergene may consist mostly of regulatory genes without a previously identified role in 208 shell biosynthesis. However, two sets of transcripts could be putatively linked to shell or 209 pigment production.

211 The first set consists of transcripts involved in the synthesis of the nacreous layer in the 212 snail's shell. Mollusc shells consists of three layers: the outer prismatic layer, the inner 213 prismatic layer and the nacreous layer (Suzuki \& Nagasawa, 2013). It is thought that

214 dermatopontin, a major shell matrix protein, is involved in the production of the nacreous 215 layer (Jiao et al., 2012). We found two hits to the Euhadra herklotsi ortholog of this gene.

216 Two other transcripts showed resemblance to Mucin 2, which is a gene that has a mollusc 217 homolog that is thought to be involved with production of the nacreous layer in molluscs 218 (Marin et al., 2000). The mollusc variant of this gene is nacroperlin, which is found in shell 
219 of Mediterranean mussels (Marin et al., 2000). The nacreous layer is the innermost layer of

220 the snail's shell and is therefore unlikely to directly affect pigmentation, however

221 differences in the nacreous layer may affect other shell traits that differ between the

222 morphs. Shell strength is known to differ between C. nemoralis colour morphs, with pink

223 shells stronger than yellow shells and banding stronger than no banding (Jiao et al., 2012;

224 Rosin et al., 2013).

225

226 The second set of transcripts consists of transcripts that produce metallothionein.

227 Metallothionein is a lightweight thiol-rich protein, the production of which is induced by 228 the presence of heavy metals such as zinc, copper or cadmium. This molecule inhibits the 229 production of melanin following oxidative stress (Sasaki et al., 2004). Melanin is a well230 known pigment that is found in many tissue types and often has a black or brown colour.

231 An inhibition of the production of melanin can possibly lead to a lack of pigmentation in the 232 tissue. This might contribute to the banding pattern or the ground colour of the shell.

233 Melanin pigments are produced in organelles called melanocytes, which contain a 234 subcellular zinc reservoir. This zinc can trigger a reaction with metallothionein to reduce 235 the production of melanin (Borovanský, 1994). The density of melanocytes in the snail 236 mantle was found to be correlated with darkness of the lip and possibly the banding 237 pattern on the shells (Emberton, 1963). The ground colour of the shell was not correlated 238 with the density of melanocytes. This suggests that the metallothionein transcripts we 239 identified could be involved in the production of the banding pattern and that they are less 240 likely to be involved in the ground colour. 
242 The aim of this research was to find candidate genes underpinning the Cepaea shell colour

243 polymorphism. Due to the modest sample size, our power to detect differential expression

244 was limited. Furthermore, we found a relatively high ratio of synonymous to

245 nonsynonymous SNPs (close to 1:1), which was probably the result of over-prediction of

246 coding regions in partial transcripts and transcripts overlapping introns. This can result in

247 a higher number of predicted non-synonymous SNPS (Lopez-Maestre et al., 2016).

248 Nevertheless, we identified 300 candidates that showed mantle-specific expression and

249 phenotype-consistent SNP patterns. In addition to these, we found fifteen transcripts

250 matching RAD-tag sequences that are associated with the shell-colour supergene.

251 Functional annotation of these transcripts should be an excellent starting point for

252 elucidating the molecular underpinning on the Cepaea colour polymorphism.

\section{Acknowledgements}

255 We thank Heike Kappes for performing the snail dissections, Emile de Meijer and Henk

256 Buermans for RNA extraction and library preparation, and Peter Neleman, Mirna Baak and 257 Patrick Wijntjes for their earlier analysis of this dataset.

258

259 Animal Ethics

260 Not applicable

261

262 DNA deposition 
263 The sequencing data has been deposited to NCBI's Sequence Read Archive (SRA) under

264 accession SRP101411. The assembled transcriptome has been submitted to NCBI

265 transcriptome shotgun assembly database (TSA) under BioProject No. PRJNA377398.

266

\section{Supplemental Information}

268 Figure S1 Snails used in this study.

269 Figure S2 Frequency distribution of the number of transcripts per gene.

270 Data S1 GO-annotation results

271 Data S2 Differential expression analysis results

272 Data S3 Hits and alignments of blasting the RAD-tags to the reference transcriptome.

273

274 References

275 Altschul SF., Gish W., Miller W., Myers EW., Lipman DJ. 1990. Basic local alignment search

276 tool. J. Mol. Biol. 215:403-410. DOI: 10.1016/S0022-2836(05)80360-2.

277 Arnold RW. 1971. Heredity - Abstract of article: Cepaea nemorlis on the east sussex south

278 Downs, and the nature of area effects. Heredity 26:277-298.

279 Borovanský J. 1994. Zinc in pigmented cells and structures, interactions and possible roles. 280 Sborník Lékařský 95:309-320.

281 Cain AJ., Sheppard PM. 1950. Selection in the polymorphic land snail Cepaea nemoralis. $282 \quad$ Heredity $4: 275-294$.

283 Cain A., Sheppard P. 1952. The effects of natural selection on body colour in the land snail 284 Cepaea nemoralis. Heredity 6:217-231.

285 Cain AJ., Sheppard PM. 1954. Natural Selection in Cepaea. Genetics 39:89-116. 
286 Camacho C., Coulouris G., Avagyan V., Ma N., Papadopoulos J., Bealer K., Madden TL. 2009.

287 BLAST+: architecture and applications. BMC Bioinformatics 10:421. DOI:

$288 \quad 10.1186 / 1471-2105-10-421$.

289 Cameron RAD., Cook LM. 2012. Habitat and the shell polymorphism of Cepaea nemoralis

290 (L.): Interrogating the Evolution Megalab database. Journal of Molluscan Studies

291 78:179-184. DOI: 10.1093/mollus/eyr052.

292 Cingolani P., Platts A., Wang LL., Coon M., Nguyen T., Wang L., Land SJ., Ruden DM., Lu X.

293 2012. A program for annotating and predicting the effects of single nucleotide

294 polymorphisms, SnpEff: SNPs in the genome of Drosophila melanogaster strain

295 w1118; iso-2; iso-3. Fly 6:1-13.

296 Clarke B. 1962. Heredity - Abstract of article: Natural selection in mixed populations of two 297 polymorphic snails. Heredity 17:319-345.

298 Cook LM. 1998. A two-stage model for Cepaea polymorphism. Philosophical Transactions of 299 the Royal Society B: Biological Sciences 353:1577-1593. DOI: 10.1098/rstb.1998.0311.

300 Emberton LRB. 1963. Relationships Between Pigmentation of Shell and of Mantle in the 301 Snails Cepaea Nemoralis (1.) and Cepaea Hortensis (mull.). Proceedings of the 302 Zoological Society of London 140:273-293. DOI: 10.1111/j.1469-7998.1963.tb01864.x.

303 Fu L., Niu B., Zhu Z., Wu S., Li W. 2012. CD-HIT: Accelerated for clustering the next-

304 generation sequencing data. Bioinformatics 28:3150-3152. DOI:

$305 \quad$ 10.1093/bioinformatics/bts565.

306 Grabherr MG., Haas BJ., Yassour M., Levin JZ., Thompson DA., Amit I., Adiconis X., Fan L., 307 Raychowdhury R., Zeng Q., Chen Z., Mauceli E., Hacohen N., Gnirke A., Rhind N., di

308 Palma F., Birren BW., Nusbaum C., Lindblad-Toh K., Friedman N., Regev A. 2011. 
Trinity: reconstructing a full-length transcriptome without a genome from RNA-Seq data. Nature biotechnology 29:644-652. DOI: 10.1038/nbt.1883.

311 Greenwood J. 1974. Visual and other selection in Cepaea: A further example. Heredity $33: 17-31$.

Jiao Y., Wang H., Du X., Zhao X., Wang Q., Huang R., Deng Y. 2012. Dermatopontin, a shell matrix protein gene from pearl oyster Pinctada martensii, participates in nacre formation. Biochemical and Biophysical Research Communications 425:679-683. DOI: 10.1016/j.bbrc.2012.07.099.

Jones JS., Leith BH., Rawlings P. 1977. Polymorphism in Cepaea: a problem with too many solutions? Annual Review of Ecology and Systematics 8:109-143.

Lamotte M. 1959. Polymorphism of Natural Populations of Cepaea nemoralis. Cold Spring Harbor Symposia on Quantitative Biology 24:65-86. DOI: 10.1101/SQB.1959.024.01.009.

Langmead B., Salzberg SL. 2012. Fast gapped-read alignment with Bowtie 2. Nature methods 9:357-9. DOI: 10.1038/nmeth.1923. without a reference genome: identification, quantification, differential analysis and impact on the protein sequence. Nucleic Acids Res. 44, e148-e148. the common grove snail Cepaea nemoralis. BMC Genomics 15:249. DOI: 10.1186/14712164-15-249.

Marin F., Corstjens P., Gaulejac B de., Jong E de V-D., Westbroek P. 2000. Mucins and 
molluscan calcification. Molecular characterization of mucoperlin, a novel mucin-like protein from the nacreous shell layer of the fan mussel Pinna nobilis (Bivalvia, pteriomorphia). Journal of Biological Chemistry 275:20667-20675. DOI: 10.1074/jbc.M003006200.

McKenna A., Hanna M., Banks E., Sivachenko A., Cibulskis K., Kernytsky A., Garimella K., Altshuler D., Gabriel S., Daly M., DePristo M a. 2010. The Genome Analysis Toolkit: a MapReduce framework for analyzing next-generation DNA sequencing data. Genome research 20:1297-303. DOI: 10.1101/gr.107524.110.

Murray J. 1963. The Inheritance of Some Characters in Cepaea Hortensis and Cepaea

\section{2} Nemoralis (Gastropoda). Genetics 48:605-615.

Richards PM., Liu MM., Lowe N., Davey JW., Blaxter ML., Davison A. 2013. RAD-Seq derived markers flank the shell colour and banding loci of the Cepaea nemoralis supergene.

Roberts A., Pachter L. 2013. Streaming fragment assignment for real-time analysis of Molecular Ecology 22:3077-3089. DOI: 10.1111/mec.12262.

Rosin ZM., Kobak J., Lesicki A., Tryjanowski P. 2013. Differential shell strength of Cepaea

Robinson MD., McCarthy DJ., Smyth GK. 2010. edgeR: a Bioconductor package for sequencing experiments. Nature Methods 10:71-73. DOI: 10.1038/nmeth.2251. nemoralis colour morphs--implications for their anti-predator defence. Die Naturwissenschaften 100:843-851. DOI: 10.1007/s00114-013-1084-8.

Sasaki M., Kizawa K., Igarashi S., Horikoshi T., Uchiwa H., Miyachi Y. 2004. Suppression of 354 melanogenesis by induction of endogenous intracellular metallothionein in human 
melanocytes. Experimental Dermatology 13:465-471. DOI: 10.1111/j.0906-

356 6705.2004.00204.x.

357 Salazar-Jaramillo L., Jalvingh KM., de Haan A., Kraaijeveld K., Buermans H., Wertheim B.

358 2017. Inter- and intra-species variation in genome-wide gene expression of Drosophila

359 in response to parasitoid wasp attack. BMC Genomics 18:331. DOI: 10.1186/s12864-

360 017-3697-3.

361 Schilthuizen M. 2013. Rapid, habitat-related evolution of land snail colour morphs on 362 reclaimed land. Heredity 110:247-52. DOI: 10.1038/hdy.2012.74.

363 Schwander T., Libbrecht R., Keller L. 2014. Supergenes and complex phenotypes. Current 364 Biology 24:R288-R294. DOI: 10.1016/j.cub.2014.01.056.

365

366

367

368

369

370

371

372

373 374 375 376 377

Silvertown J., Cook L., Cameron R., Dodd M., McConway K., Worthington J., Skelton P., Anton C., Bossdorf O., Baur B., Schilthuizen M., Fontaine B., Sattmann H., Bertorelle G., Correia M., Oliveira C., Pokryszko B., Ozgo M., Stalažs A., Gill E., Rammul Ü., Sólymos P., Féher Z., Juan X. 2011. Citizen science reveals unexpected continental-scale evolutionary change in a model organism. PLoS ONE 6. DOI: 10.1371/journal.pone.0018927.

Simao FA., Waterhouse RM., Ioannidis P., Kriventseva E V., Zdobnov EM. 2015. BUSCO: Assessing genome assembly and annotation completeness with single-copy orthologs. Bioinformatics 31:3210-3212. DOI: 10.1093/bioinformatics/btv351.

Suzuki M., Nagasawa H. 2013. Mollusk shell structures and their formation mechanism. Canadian Journal of Zoology 91:349-366. DOI: 10.1139/cjz-2012-0333.

Worthington JP., Silvertown J., Cook L., Cameron R., Dodd M., Greenwood RM., McConway K., Skelton P. 2012. Evolution MegaLab: a case study in citizen science methods. Methods in Ecology and Evolution 3:303-309. DOI: 10.1111/j.2041- 
378 210X.2011.00164.x.

379

380

381 
382 383

384

385

386

387

388

389

390

391

392

393

394

395

396

397

398

399

\begin{tabular}{lcc}
\hline Property & Number of SNPS & $\begin{array}{c}\text { Number of } \\
\text { transcripts }\end{array}$ \\
\hline Total number & 73,817 & 17,499 \\
Consistent & 5,776 & 2,592 \\
Differentially Expressed & 4,992 & 817 \\
Differentially expressed and & 569 & 197 \\
consistent & & \\
\hline
\end{tabular}

Table 1 Overview of number of reads and GC content per sample and tissue.

\begin{tabular}{cccccc}
\hline Sample & Individual & Tissue & Number of reads & Number of bases & GC Content \\
\hline 11 & 1 & Foot & $71,694,188$ & $6,273,241,450$ & $44 \%$ \\
12 & 1 & Mantle & $71,670,338$ & $6,271,154,575$ & $46 \%$ \\
13 & 2 & Foot & $77,053,092$ & $6,742,145,550$ & $43 \%$ \\
14 & 2 & Mantle & $66,729,632$ & $5,838,842,800$ & $46 \%$ \\
15 & 3 & Foot & $63,903,422$ & $5,591,549,425$ & $46 \%$ \\
16 & 3 & Mantle & $69,572,264$ & $6,087,573,100$ & $49 \%$ \\
17 & 4 & Foot & $121,731,962$ & $10,651,546,675$ & $46 \%$ \\
18 & 4 & Mantle & $139,455,234$ & $12,202,332,975$ & $49 \%$ \\
\hline
\end{tabular}

Table 2 Statistics of the transcriptome assembly.

\begin{tabular}{lcc}
\hline Statistic & Number & Number after filtering \\
\hline Number of contigs & 171,051 & 147,411 \\
Average contig length & $847.86 \mathrm{bp}$ & $783.47 \mathrm{bp}$ \\
Median contig length & $537 \mathrm{bp}$ & $515 \mathrm{bp}$ \\
Number of genes & 33,109 & 25,334 \\
N50 & 1,111 & 968 \\
GC Content & $42.20 \%$ & $42 \%$ \\
Total bases & $145,027,740$ & $115,481,543$ \\
\hline
\end{tabular}

Table 3 Numbers of SNPs that were homozygous in the yellow snails and the number of transcripts these were found in. These are further broken down into sets that showed allelic patterns that were consistent with the shell phenotypes of the sampled snails, differentially expressed in mantle tissue or both.
400

401

402 
Table 4 The most informative annotations from Supplementary Table S3. Transcripts with 408

409

410 putative function in nacre and shell production are marked in italics.

\begin{tabular}{|c|c|c|}
\hline Contig name & Functional annotation & Effect \\
\hline c280576_g1_i1 & $\begin{array}{l}\text { Biomphalaria glabrata glycine and } \\
\text { methionine-rich -like }\end{array}$ & synonymous_variant \\
\hline c280925_g1_i2 & $\begin{array}{l}\text { Parasteatoda tepidariorum keratin- } \\
\text { associated 6-2-like }\end{array}$ & synonymous_variant \\
\hline c350256_g1_i1 & $\begin{array}{l}\text { Anas platyrhynchos BPI fold- } \\
\text { containing family B member } 3\end{array}$ & synonymous_variant \\
\hline c368572_g1_i1 & $\begin{array}{l}\text { Aplysia californica epithelial } \\
\text { splicing regulatory } 1 \text {-like }\end{array}$ & synonymous_variant \\
\hline c369765_g4_i2 & $\begin{array}{l}\text { Biomphalaria glabrata ferric- } \\
\text { chelate reductase } 1 \text {-like }\end{array}$ & synonymous_variant \\
\hline c371799_g2_i1 & $\begin{array}{l}\text { Biomphalaria glabrata sushi, von } \\
\text { Willebrand factor type A, EGF and } \\
\text { pentraxin domain-containing } 1 \text { - } \\
\text { like }\end{array}$ & synonymous_variant \\
\hline c264073_g1_i1 & Camelus bactrianus mucin-2-like & $\begin{array}{l}\text { frameshift_variant \& } \\
\text { stop_gained } \\
\text { inframe_insertion }\end{array}$ \\
\hline c366293_g1_i1 & Biomphalaria glabrata mucin-2-like & missense_variant \\
\hline c321814_g1_i1 & $\begin{array}{l}\text { Euhadra herklotsi mRNA for } \\
\text { Dermatopontin1 }\end{array}$ & intergenic_region \\
\hline c355427_g1_i1 & $\begin{array}{l}\text { Euhadra herklotsi mRNA for } \\
\text { Dermatopontin1 }\end{array}$ & $\begin{array}{l}\text { stop_gained } \\
\text { missense_variant }\end{array}$ \\
\hline
\end{tabular}




\section{Figure legends}

413 Figure 1 Overall gene expression differences according to tissue and individual snail.

414 Figure 2 Scatterplot (A):log counts versus log fold change and volcano plot (B): log fold

415 change versus statistical significance, for differential expression in mantle tissue versus the 416 foot tissue, obtained using EdgeR. Transcripts marked in red were considered differentially 417 expressed.

418

419

420 


\section{Figure 1}

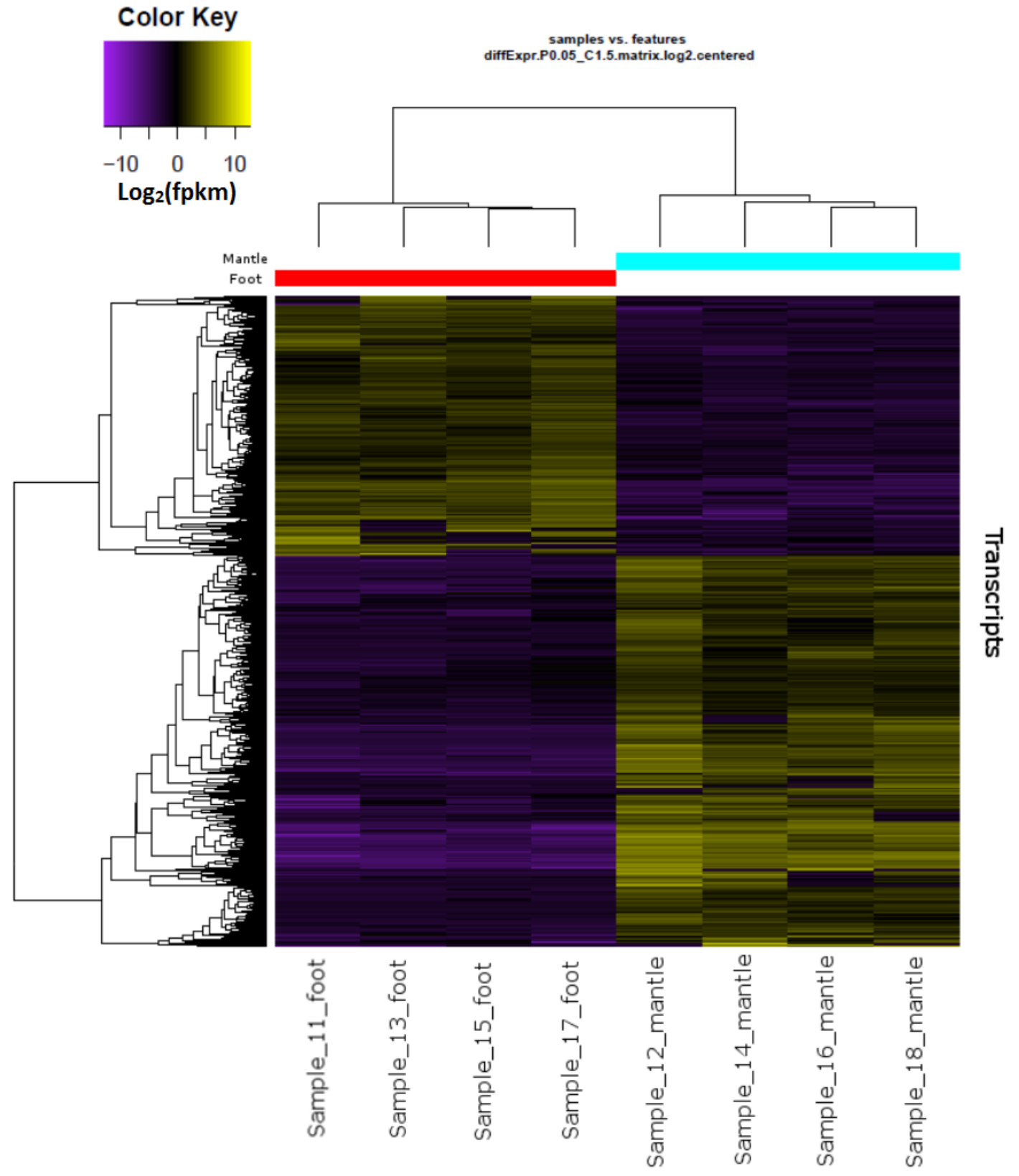




\section{Figure 2}

A)

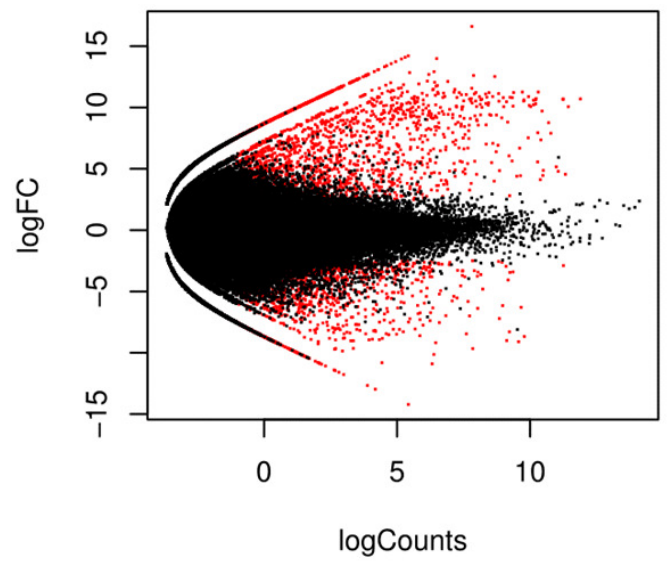

B)

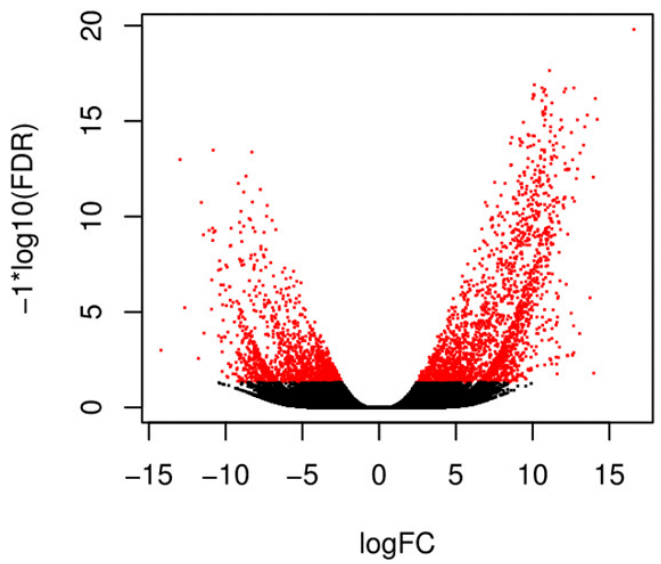

426

427

428 